\title{
Conditional stability of unstable viscous shocks
}

\author{
Kevin ZuMBrun*
}

November 2, 2018

\begin{abstract}
Continuing a line of investigation initiated by Texier and Zumbrun on dynamics of viscous shock and detonation waves, we show that a linearly unstable Lax-type viscous shock solution of a semilinear strictly parabolic system of conservation laws possesses a translationinvariant center stable manifold within which it is nonlinearly orbitally stable with respect to small $L^{1} \cap H^{2}$ perturbatoins, converging timeasymptotically to a translate of the unperturbed wave. That is, for a shock with $p$ unstable eigenvalues, we establish conditional stability on a codimension- $p$ manifold of initial data, with sharp rates of decay in all $L^{p}$. For $p=0$, we recover the result of unconditional stability obtained by Howard, Mascia, and Zumbrun.
\end{abstract}

\section{Contents}

1 Introduction $\quad 2$

1.1 Discussion and open problems ............ . . 5

2 Center Stable Manifold for ODE 5

2.1 Frechet differentiability of substitution operators . . . . . . 6

2.2 Smooth dependence of fixed-point solutions . . . . . . . . 7

2.3 Global Center Stable Manifold construction . . . . . . . . . 9

2.4 Local construction: proof of Theorem 2.1 . . . . . . . . . 11

*Indiana University, Bloomington, IN 47405; kzumbrun@indiana.edu: Research of K.Z. was partially supported under NSF grants no. DMS-0070765 and DMS-0300487. 
3 Center Stable Manifold for PDE 12

3.1 Reduced equations . . . . . . . . . . . . . . . . . 13

3.2 Preliminary estimates . . . . . . . . . . . . . . . . 15

3.3 Translation-invariant center stable manifold . . . . . . . . 16

3.4 Application to viscous shock waves . . . . . . . . . . 17

4 Conditional stability analysis 18

4.1 Projector bounds . . . . . . . . . . . . . . . . . 18

4.2 Linear estimates . . . . . . . . . . . . . . . . . . . . . . . . . 19

4.3 Reduced equations II . . . . . . . . . . . . . . . . . . . 22

4.4 Nonlinear damping estimate . . . . . . . . . . . . . . . 23

4.5 Proof of nonlinear stability . . . . . . . . . . . . 24

A Proofs of miscellaneous lemmas $\quad 27$

\section{Introduction}

In this paper, we continue a line of investigation opened in [TZ1, TZ2, TZ3, TZ4, SS, BeSZ] going beyond simple stability analysis to study nontrivial dynamics, and associated physical phenomena, of perturbed viscous shock waves in the presence of linear instability. The above-mentioned references concern Hopf bifurcation to time-periodic behavior associated with transition to linear instability arising through the passage from stable to unstable halfplane of a complex conjugate pair of eigenvalues of the linearized operator about the wave. See also [ZH, Z1] for discussion of (nonstandard, due to embedding in essential spectrum) bifurcations associated with the passage of a simple eigenvalue through zero.

In the present work, in the spirit of [GJLS, Li] and other works outside the shock wave context, we consider the situation of a viscous shock substantially after the onset of instability, i.e., with one or more strictly unstable but no neutrally unstable eigenvalues, and seek to describe the nearby phase portrait in terms of invariant manifolds and behavior therein. Specifically, for shock waves of systems of conservation laws with artificial viscosity, we construct a center stable manifold and show that the shock is conditionally (nonlinearly) stable with respect to this codimension $p$ set of initial data, where $p$ is the number of unstable eigenvalues. As discussed for example in 
[AMPZ, GZ], such conditionally stable shock waves can play an important role in asymptotic behavior as metastable states.

Consider a viscous shock solution $u(x, t)=\bar{u}(x), \lim _{z \rightarrow \pm \infty} \bar{u}(z)=u_{ \pm}$, without loss of generality stationary, of a semilinear parabolic system of conservation laws

$$
u_{t}+f(u)_{x_{j}}=u_{x x},
$$

$u, f \in \mathbb{R}^{n}, x, t \in \mathbb{R}$, under the basic assumptions:

(H0) $f \in C^{k+2}, k \geq 2$.

(H1) $A_{ \pm}:=d f\left(u_{ \pm}\right)$have simple, real, nonzero eigenvalues.

Linearizing (1.1) about $\bar{u}$ yields linearized equations

$$
u_{t}=L u:=-(d f(\bar{u}) u)_{x}-u_{x x},
$$

for which the generator $L$ possesses $[\mathrm{He}, \mathrm{S}, \mathrm{ZH}]$ both a translational zeroeigenvalue and essential spectrum tangent at zero to the imaginary axis.

The absence of a spectral gap between neutral (i.e., zero real part) and stable (negative real part) spectra of $L$ prevents the usual ODE-type decomposition of the flow near $\bar{u}$ into invariant stable, center, and unstable manifolds. The first result of this paper, by now little more than a remark, is that we can still determine center stable and unstable manifolds, and that these may be chosen to respect the underlying translation-invariance of (1.1). See [TZ1] for closely related results on existence of translational-invariant center manifolds. As the needed ingredients do not seem to be found in one place, we nonetheless for completeness carry out the proof in full detail.

Theorem 1.1. Under assumptions (HO)-(H1), there exists in an $H^{2}$ neighborhood of the set of translates of $\bar{u}$ a codimension-p translation invariant $C^{k}$ (with respect to $H^{2}$ ) center stable manifold $\mathcal{M}_{c s}$, tangent at $\bar{u}$ to the center stable subspace $\Sigma_{c s}$ of $L$, that is (locally) invariant under the forward time-evolution of (3.1) and contains all solutions that remain bounded and sufficiently close to a translate of $\bar{u}$ in forward time, where $p$ is the (necessarily finite) number of unstable, i.e., positive real part, eigenvalues of $L$.

Next, specializing a bit further, we add to (H0)-(H1) the additional hypothesis that $\bar{u}$ be a Lax-type shock:

(H2) The dimensions of the unstable subspace of $d f\left(u_{-}\right)$and the stable subspace of $d f\left(u_{+}\right)$sum to $n+1$. 
We assume further the following spectral genericity conditions.

(D1) $L$ has no nonzero imaginary eigenvalues.

(D2) The orbit $\bar{u}(\cdot)$ is a transversal connection of the associated standing wave equation $\bar{u}_{x}=f(\bar{u})-f\left(u_{-}\right)$.

(D3) The associated inviscid shock $\left(u_{-}, u_{+}\right)$is hyperbolically stable, i.e.,

$$
\operatorname{det}\left(r_{1}^{-}, \ldots, r_{p-1}^{-}, r_{p+1}^{+}, \ldots, r_{n}^{+},\left(u_{+}-u_{-}\right)\right) \neq 0,
$$

where $r_{1}^{-}, \ldots r_{p-1}^{-}$denote eigenvectors of $d f\left(u_{-}\right)$associated with negative eigenvalues and $r_{p+1}^{+}, \ldots r_{n}^{+}$denote eigenvectors of $d f\left(u_{+}\right)$associated with positive eigenvalues.

As discussed in [ZH, MaZ1], (D2)-(D3) correspond in the absence of a spectral gap to a generalized notion of simplicity of the embedded eigenvalue $\lambda=0$ of $L$. Thus, (D1)-(D3) together correspond to the assumption that there are no additional (usual or generalized) eigenvalues on the imaginary axis other than the transational eigenvalue at $\lambda=0$; that is, the shock is not in transition between different degrees of stability, but has stability properties that are insensitive to small variations in parameters.

With these assumptions, we obtain our second and main result characterizing stability properties of $\bar{u}$. In the case $p=0$, this reduces to the nonlinear orbital stability result established in [ZH, MaZ1, MaZ2, MaZ3, Z2, Z3, Z4].

Theorem 1.2. Under (HO)-(H2) and (D1)-(D3), $\bar{u}$ is nonlinearly orbitally stable under sufficiently small perturbations in $L^{1} \cap H^{4}$ lying on the codimension $p$ center stable manifold $\mathcal{M}_{c s}$ of $\bar{u}$ and its translates, where $p$ is the number of unstable eigenvalues of $L$, in the sense that, for some $\alpha(\cdot)$,

$$
\begin{aligned}
|u(x, t)-\bar{u}(x-\alpha(t))|_{L^{p}} & \leq C(1+t)^{-\frac{1}{2}\left(1-\frac{1}{p}\right)}|u(x, 0)-\bar{u}(x)|_{L^{1} \cap H^{4}}, \\
|u(x, t)-\bar{u}(x-\alpha(t))|_{H^{4}} & \leq C(1+t)^{-\frac{1}{4}}|u(x, 0)-\bar{u}(x)|_{L^{1} \cap H^{4}}, \\
\dot{\alpha}(t) & \leq C(1+t)^{-\frac{1}{2}}|u(x, 0)-\bar{u}(x)|_{L^{1} \cap H^{4}}, \\
\alpha(t) & \leq C|u(x, 0)-\bar{u}(x)|_{L^{1} \cap H^{4}}
\end{aligned}
$$

Moreover, it is orbitally unstable with respect to small $\mathrm{H}^{2}$ perturbations not lying in $\mathcal{M}$, in the sense that the corresponding solution leaves a fixed-radius neighborhood of the set of translates of $\bar{u}$ in finite time. 


\subsection{Discussion and open problems}

It is easily checked that the results of this paper go through for general semilinear parabolic term $B u_{x x}, B$ constant, under the standard assumptions of $[\mathrm{ZH}]$. An interesting open problem is to extend these results to the quasilinear and or partially parabolic ("real viscosity") case. We note that the only issue here is to establish existence of the center stable manifold, as the proof of stability goes through essentially unchanged, incorporating the necessary modifications detailed in [Z2, Z3] to deal with the quasilinear/partially parabolic case. See also related discussion in [Li].

Another interesting problem would be to extend our conditional stability result to the case of nonclassical under- or overcompressive shocks using pointwise estimates as in [HZ, RZ]; see Remark 4.7.

Finally, we mention the problem of determining conditional stability of a planar standing shock $u(x, t) \equiv \bar{u}\left(x_{1}\right)$ of a multidimensional system of conservation laws

$$
u_{t}+\sum_{j} f_{j}(u)_{x_{j}}=\Delta_{x} u
$$

which likewise (by the multidimensional arguments of [Z1, Z2, Z3]) reduces to construction of a center stable manifold, in this case involving an infinitedimensional unstable subspace corresponding to essential spectra of the linearized operator $L$ about the wave.

Plan of the paper. In Section 2 we give for completeness a particularly concise proof of the center stable manifold theorem for ODE. In Section 3, loosely following [TZ1], we show how to extend this to semilinear parabolic PDE, while preserving the key property of translation-invariance. Finally, in Section 4, we establish conditional stability by a modification of the arguments of $[\mathrm{Z} 4, \mathrm{MaZ2}, \mathrm{MaZ3}]$ in the stable $(p=0)$ case.

\section{Center Stable Manifold for ODE}

The center stable manifold construction in the PDE case follows closely the construction for finite-dimensional ODE, which we therefore recall here for completeness; see also [B, VI]. Consider an ODE

$$
u^{\prime}=f(u), \quad f \in C^{1},
$$


and an equilibrium $f\left(u_{*}\right)=0$, with associated linearized equation

$$
v^{\prime}=A v, \quad A:=d f\left(u_{*}\right) .
$$

Associated with $A$, define the center stable subspace $\Sigma_{c s}$ as the direct sum of all eigenspaces of $A$ associated to neutral or stable eigenvalues, i.e., eigenvalues with zero or positive real part. Likewise, define the unstable subspace $\Sigma_{u}$ as the direct sum of eigenspaces associated to unstable eigenvalues, i.e., eigenvalues with strictly positive real part, so that $\mathbb{R}^{n}=\Sigma_{c s} \oplus \Sigma_{u}$.

Defining the associated (total) eigenprojections $\Pi_{c s}$ and $\Pi_{u}$ as the sum of all eigenprojections associated with neutral-stable and unstable eigenvalues, respectively, we have, either by reduction to Jordan form or direct estimation using the inverse Laplace transform/resolvent estimates, bounds

$$
\begin{aligned}
\left|e^{A t} \Pi_{c s}\right| & \leq C(\eta) e^{\theta t} \quad t \geq 0, \\
\left|e^{A t} \Pi_{u}\right| & \leq C(\theta) e^{-\eta|t|}, \quad t \leq 0,
\end{aligned}
$$

for any $\eta>0$ strictly smaller than the minimum of the real parts of unstable eigenvalues and $\theta>0$ arbitrarily small.

Proposition 2.1 (Center Stable Manifold Theorem for ODE). For $f$ in $C^{k+1}, 1 \leq k<\infty$, there exists local to $u_{*}$ a $C^{k}$ center stable manifold $\mathcal{M}_{c s}$, tangent at $u_{*}$ to $\Sigma_{c s}$, expressible in coordinates $w:=u-u_{*}$ as a $C^{k}$ graph $\Phi_{c s}: \Sigma_{c s} \rightarrow \Sigma_{c s} \oplus \Sigma_{u}$, that is (locally) invariant under the flow of (2.1) and contains all solutions that remain bounded and sufficiently close to $u_{*}$ in forward time. In general it is not unique.

\subsection{Frechet differentiability of substitution operators}

Standard invariant (e.g., stable, center, center stable) manifold constructions proceed by fixed point iteration on various weighted $L^{\infty}$ spaces

$$
\|f\|_{\eta}:=\sup _{t \geq t_{0}} e^{\eta\left(t-t_{0}\right)}|f(t)|, \quad \mathcal{B}_{\eta}:=\left\{f:\|f\|_{\eta}<\infty\right\}
$$

with $\eta$ positive for stable manifolds and negative for center or center stable manifolds. As described in [B, VI], Frechet differentiability of the associated fixed-point mapping (hence eventual smoothness of the resulting manifold) hinges on Frechet differentiability with respect to spaces $\mathcal{B}_{\eta}$ of the special class of substitution operators, defined for $g: \mathbb{R}^{n} \rightarrow \mathbb{R}^{n}$ as

$$
G(f)(t):=g(f(t)) .
$$


Let $C_{b}^{k}$ denote the Banach space of $C^{k}$ functions $g: \mathbb{R}^{n} \rightarrow \mathbb{R}^{n}$ with $\left|d^{j} g\right|$ uniformly bounded for $0 \leq j \leq k$, with associated norm

$$
\|g\|_{C_{b}^{k}}:=\sum_{0 \leq j \leq k} \sup _{\mathbb{R}^{n}}\left|d^{j} g\right| .
$$

Lemma 2.2. For $\eta \geq 0, k \geq 1$, if $g \in C_{b}^{k}\left(\mathbb{R}^{n} \rightarrow \mathbb{R}^{n}\right)$, then $G$ is $C^{k}$ from $\mathcal{B}_{\eta} \rightarrow \mathcal{B}_{\eta}$, with $d^{k} G f(t)=\left(d^{k} g\right)(f(t))$.

Proof. See Appendix A.

Lemma 2.3. Let $g \in C_{b}^{k+1}$ and $0<-\eta^{\prime}<-\eta / k$. Then, the substitution operator $G$ is $C^{k}$ from $\mathcal{B}_{\eta^{\prime}} \rightarrow \mathcal{B}_{\eta}$, with $d^{k} G f(t)=\left(d^{k} g\right)(f(t))$.

Proof. More generally, the result of Lemma 2.3 holds for any $g \in C_{b}^{k+\alpha}$, $0<\alpha \leq 1$, in the sense that $\left|d^{k} g(x+h)-d^{k} g(x)\right| \leq C|h|^{\alpha}$ for some uniform $C>0$, as may be seen by rewriting the $(k-1)$ th-order Taylor remainder formula $g(x+h)-\mathcal{T}_{k-1} g(x, h)=\left(\int_{0}^{1} d^{k} g(x+\theta h) \frac{(1-\theta)^{k-1}}{(k-1) !} d \theta\right) h^{k}$ as

$$
g(x+h)-\mathcal{T}_{k} g(x, h)=\left(\int_{0}^{1}\left(d^{k} g(x+\theta h)-d^{k} g(x)\right) \frac{(1-\theta)^{k-1}}{(k-1) !} d \theta\right) h^{k},
$$

where $\mathcal{T}_{k} g(x, h)$ denotes the $k$ th-order Taylor expansion of $g$ about $x$ evaluated at $x+h$, then using the assumed uniform bound on $\left|d^{k} g(x+h)-d^{k} g(x)\right|$ to obtain $\left|g(x+h)-\mathcal{T}_{k} g(x, h)\right| \leq C|h|^{k+\alpha}$, and thus

$$
\left|g(x+h)-\mathcal{T}_{k} g(x, h)\right|_{-(k+\alpha) \eta^{\prime}} \leq C|h|_{\eta^{\prime}}^{k+\alpha} .
$$

Without loss of generality taking $\alpha$ sufficiently small, this yields the result. A similar estimate yields continuity of the $k$ th Frechet derivative.

\subsection{Smooth dependence of fixed-point solutions}

We next present two general results on smooth dependence of fixed point solutions. Let $T(x, y)$ be continuous in $x, y$ and contractive in $y, T: \mathcal{B}_{1} \times$ $\mathcal{B}_{2} \rightarrow \mathcal{B}_{2}$ for Banach spaces $\mathcal{B}_{1}$ and $\mathcal{B}_{2}$, defining a fixed point map $y(x)$, $y: \mathcal{B}_{1} \rightarrow \mathcal{B}_{2}$, continuous in the parameter $x$, such that $y(x)=T(x, y(x))$. Then, we have the following standard result. 
Lemma 2.4. If $T$ is Lipschitz in $(x, y)$, then $y$ is Lipschitz from $\mathcal{B}_{1} \rightarrow \mathcal{B}_{2}$. If $T$ is $C^{k}$ (Frechet sense), $k \geq 1$, in $(x, y)$, then $y$ is $C^{k}$ from $\mathcal{B}_{1} \rightarrow \mathcal{B}_{2}$, with

$$
(d y / d x)\left(x_{0}\right)=\left(\operatorname{Id}-T_{y}\right)^{-1} T_{x}\left(x_{0}, y\left(x_{0}\right)\right)
$$

and higher derivatives $\left(d^{j} y / d x^{j}\right)\left(x_{0}\right), 1 \leq j \leq k$ obtained by formal differentiation of (2.6), substituting for lower derivatives wherever they appear.

Proof. See Appendix A.

The next Lemma shows how we can recover $C^{1}$ dependence of fixed point solutions in the case, as in Lemma 2.3, that $T$ is differentiable only from a stronger to a weaker space. We discuss higher derivatives later where they appear, since they involve specific chains of successively weaker spaces that are not convenient for statement as a general theorem. Let $\mathcal{B}_{2}^{\prime} \subset \mathcal{B}_{2}$, with $\|\cdot\|_{\mathcal{B}_{2}^{\prime}} \geq\|\cdot\|_{\mathcal{B}_{2}}$, and $T(x, y)$ be a map $\mathcal{B}_{1} \times \mathcal{B}_{2}^{\prime} \rightarrow \mathcal{B}_{2}^{\prime}$ that is Lipschitz continuous in $(x, y)$ and contractive (with respect to $\|\cdot\|_{\mathcal{B}_{2}^{\prime}}$ ) in $y$. Denote by $y(x): \mathcal{B}_{1} \rightarrow \mathcal{B}_{2}^{\prime}$ the unique Lipschitz fixed-point solution defined by $y(x)=T(x, y(x))$.

Lemma 2.5. If (i) $T$ is continuously differentiable from $\mathcal{B}_{1} \times \mathcal{B}_{2}^{\prime} \rightarrow \mathcal{B}_{2}$, and (ii) $T_{y}$ extends to a bounded linear operator from $\mathcal{B}_{2} \rightarrow \mathcal{B}_{2}$, continuous in operator norm with respect to $(x, y)$, with $\left|T_{y}\right|_{\mathcal{B}_{2}}<1$, then $y$ is continously differentiable from $\mathcal{B}_{1} \rightarrow \mathcal{B}_{2}$, with $(d y / d x)\left(x_{0}\right)=\left(\operatorname{Id}-T_{y}\right)^{-1} T_{x}\left(x_{0}, y\left(x_{0}\right)\right)$.

Proof. By Taylor's Theorem and $\left\|y\left(x_{2}\right)-y\left(x_{1}\right)\right\|_{\mathcal{B}_{2}^{\prime}} \leq L\left\|x_{2}-x_{1}\right\|_{\mathcal{B}_{1}}$, we have

$$
\begin{aligned}
y\left(x_{2}\right)-y\left(x_{1}\right)= & T_{x}\left(x_{2}-x_{1}\right)+T_{y}\left(y_{2}-y_{1}\right) \\
& +o\left(\left\|x_{2}-x_{1}\right\|_{\mathcal{B}_{1}}+\left\|y\left(x_{2}\right)-y\left(x_{1}\right)\right\|_{\mathcal{B}_{2}^{\prime}}\right) \\
= & T_{x}\left(x_{2}-x_{1}\right)+T_{y}\left(y_{2}-y_{1}\right)+o\left(\left\|x_{2}-x_{1}\right\|_{\mathcal{B}_{1}}\right),
\end{aligned}
$$

where the $o\left(\left\|x_{2}-x_{1}\right\|_{\mathcal{B}_{1}}\right)$ term is measured in the weaker $\|\cdot\|_{\mathcal{B}_{2}}$ norm. Observing by $\left|T_{y}\right|_{\mathcal{B}_{2} \rightarrow \mathcal{B}_{2}}<1$ and Neumann series inversion that (Id $-T_{y}$ ) considered as an operator from $\mathcal{B}_{2} \rightarrow \mathcal{B}_{2}$ is invertible with uniformly bounded inverse

$$
\left|\left(\mathrm{Id}-T_{y}\right)^{-1}\right|_{\mathcal{B}_{2} \rightarrow \mathcal{B}_{2}} \leq\left(1-\left|T_{y}\right|_{\mathcal{B}_{2} \rightarrow \mathcal{B}_{2}}\right)^{-1}
$$

we may solve (2.7) to obtain

$$
y\left(x_{2}\right)-y\left(x_{1}\right)=\left(\operatorname{Id}-T_{y}\right)^{-1} T_{x}\left(x_{2}-x_{1}\right)+o\left(\left\|x_{2}-x_{1}\right\|_{\mathcal{B}_{1}}\right),
$$

yielding differentiability as claimed, with $(d y / d x)=\left(\operatorname{Id}-T_{y}\right)^{-1} T_{x}$ continuous by the assumed continuity of $T_{x}$ and $T_{y}$ as operators from $\mathcal{B}_{1} \rightarrow \mathcal{B}_{1}$ and $\mathcal{B}_{2} \rightarrow \mathcal{B}_{2}$. 


\subsection{Global Center Stable Manifold construction}

We now establish a global version of 2.1 for small Lipschitz nonlinearity. As in Section 2.2, denote by $C_{b}^{k}$ the space of $C^{k}$ functions that are uniformly bounded in up to $k$ derivatives and consider for a fixed, constant matrix $A$ and an arbitrary nonlinearity $N$ such that $N(t, 0) \equiv 0, N_{w}(t, 0) \equiv 0$ the ODE

$$
w^{\prime}=A w+N(t, w) .
$$

Proposition 2.6. For $N \in C_{b}^{k+1}$ with Lipschitz constant $\varepsilon>0$ sufficiently small, (2.8) has a unique $C^{k}$ invariant manifold $\mathcal{M}_{c s}$ tangent at $w=0$ to the center stable subspace $\Sigma_{c s}$ of $A$, consisting of the union of all orbits whose solutions grow at sufficiently slow exponential rate $|w(t)| \leq C e^{\tilde{\theta}|t|}$ in positive time, for any fixed $\theta<\tilde{\theta}<\eta$.

Proof. Applying projections $\Pi_{j}, j=c s, u$ to (2.8), we obtain using the variation of constants formula equations

$$
\Pi_{j} w(t)=e^{A\left(t-t_{0, j}\right)} \Pi_{j} w\left(t_{0, j}\right)+\int_{t_{0, j}}^{t} e^{A(t-s)} \Pi_{j} N(s, w(s)) d s,
$$

$j=c s, u$, so long as the solution $w$ exists, with $t_{0, j}$ arbitrary. Assuming growth of at most $|w(t)| \leq C e^{\tilde{\theta} t}$ in positive time, we find using (2.3) and the bound $|N(w)| \leq \varepsilon|w|$ coming from $N(0)=0$ and the assumed Lipschitz bound on $N$, that as $t_{0, u} \rightarrow+\infty$, the first term $e^{A\left(t-t_{0, s}\right)} \Pi_{u} w\left(t_{0, j}\right)$ converges to zero while the second, integral term converges to $\int_{t}^{+\infty} e^{A(t-s)} \Pi_{u} N(s, w(s)) d s$, so that, choosing $t_{0, c s}=0$, we have

$$
\begin{aligned}
\Pi_{c s} w(t) & =e^{A t} \Pi_{c s} w(0)+\int_{0}^{t} e^{A(t-s)} \Pi_{c s} N(s, w(s)) d s \\
\Pi_{u} w(t) & =-\int_{t}^{+\infty} e^{A(t-s)} \Pi_{u} N(s, w(s)) d s .
\end{aligned}
$$

Summing, we obtain for $w_{c s}:=\Pi_{c s} w(0)$ the fixed-point representation

$$
\begin{aligned}
w(t)=T\left(w_{c s}, w\right):= & e^{A t} w_{c s}+\int_{0}^{t} e^{A(t-s)} \Pi_{c s} N(s, w(s)) d s \\
& -\int_{t}^{+\infty} e^{A(t-s)} \Pi_{u} N(s, w(s)) d s
\end{aligned}
$$


valid for solutions growing at rate at most $|w(t)| \leq C e^{\tilde{\theta} t}$ for $t \geq 0$.

Define now the negatively-weighted sup norm

$$
\|f\|_{-\tilde{\theta}}:=\sup _{t \geq 0} e^{-\tilde{\theta} t}|f(t)|
$$

noting that $|f(t)| \leq e^{\tilde{\theta} t}\|f\|_{-\tilde{\theta}}$ for all $t \geq 0$. We first show that, for $\left|w_{c s}\right|$ and $\delta, \varepsilon>0$ sufficiently small, the integral operator $T$ is Lipschitz in $w_{c s}$ and contractive in $w$ from the $\|\cdot\|_{-} \tilde{\theta}^{-b a l l ~} B(0, \delta)$ to itself.

Using (2.3), $|N(t, w(t))| \leq \epsilon|w(t)|$, and $|w(t)| \leq e^{\tilde{\theta}|t|}\|w\|_{-\tilde{\theta}}$, we obtain

$$
|T(w)(t)| \leq C e^{\theta|t|}\left|w_{c s}\right|+C \epsilon\|w\|_{-\tilde{\theta}}\left(\int_{0}^{t} e^{\theta|t-s|} e^{\tilde{\theta}|s|} d s+\int_{t}^{+\infty} e^{\eta(t-s)} e^{\tilde{\theta}|s|} d s\right),
$$

hence, using $\eta \pm \tilde{\theta}>0$ and taking $C_{1} \epsilon<1 / 2$ and $C\left|w_{0}\right| \leq \delta / 2$, that

$$
\begin{aligned}
\|T(w)(t)\|_{-\tilde{\theta}} \leq & C\left|w_{c s}\right|+C \epsilon\|w\|_{-\tilde{\theta}}\left(\int_{0}^{t} e^{\theta|t-s|} e^{\tilde{\theta}(|s|-|t|)} d s\right. \\
& \left.+\int_{t}^{+\infty} e^{\eta(t-s)} e^{\tilde{\theta}(|s|-|t|)} d s\right) \leq C\left|w_{c s}\right|+C_{1} \epsilon\|w\|_{-\tilde{\theta}}<\delta .
\end{aligned}
$$

Similarly, we find that

$$
\begin{aligned}
\left\|T\left(w_{1}\right)-T\left(w_{2}\right)\right\|_{-\tilde{\theta}} & \leq C \epsilon\left\|w_{1}-w_{2}\right\|_{-\tilde{\theta}}\left(\int_{0}^{t} e^{\theta|t-s|} e^{\tilde{\theta}|s|} d s+\int_{t}^{+\infty} e^{\eta(t-s)} e^{\tilde{\theta}|s|} d s\right) \\
& \leq C_{1} \varepsilon\left\|w_{1}-w_{2}\right\|_{-\tilde{\theta}}<(1 / 2)\left\|w_{1}-w_{2}\right\|_{-\tilde{\theta}}
\end{aligned}
$$

yielding contraction on $B(0, \delta)$ and thus existence of a unique fixed point $w=w\left(w_{c s}\right)$. A similar estimate shows that $T$ is Lipschitz in $w_{c s}$, so that $w(\cdot)$ is Lipshitz from $\Sigma_{c s}$ to $\mathcal{B}_{-\tilde{\theta}}$.

We next investigate smoothness of $w(\cdot)$. Note that $T$ decomposes into the sum of a bounded, hence $C^{\infty}$, linear map $w_{c s} \rightarrow e^{A t} w_{c s}$ from $\Sigma_{c s} \rightarrow \mathcal{B}_{-\tilde{\theta}}$ and the composition $\mathcal{K} \cdot \mathcal{N}$ of a bounded (hence $C^{\infty}$ ) linear map

$$
\mathcal{K}(f):=\int_{0}^{t} e^{A(t-s)} \Pi_{c s} f(s) d s-\int_{t}^{+\infty} e^{A(t-s)} \Pi_{u} f(s) d s
$$

from $\mathcal{B}_{-\tilde{\theta}} \rightarrow \mathcal{B}_{-\tilde{\theta}}$ and a substitution operator $\mathcal{N}(w)(s):=N(w(s))$ with $N \in C_{b}^{k+1}$ that by Lemma 2.3 is $C^{k}$ from $\mathcal{B}_{-\tilde{\theta} /(k+1)} \rightarrow \mathcal{B}_{-\tilde{\theta}}$. Moreover, the 
first derivative $d \mathcal{N}(w)(s)=d N(w(s))$, by $|d N| \leq \varepsilon$, extends as a bounded linear operator from $\mathcal{B}_{-\theta^{\prime}} \rightarrow \mathcal{B}_{-\theta^{\prime}}$, any $\theta^{\prime}>0$, with $|d N|_{\mathcal{B}_{-\theta^{\prime}}} \leq \varepsilon$, whence $T_{w}$ extends as a bounded linear operator from $\mathcal{B}_{-\theta^{\prime}} \rightarrow \mathcal{B}_{-\theta^{\prime}}$, that for any given $\theta^{\prime}>0$, in particular $\tilde{\theta}$, is contractive, $\left|T_{w}\right|_{\mathcal{B}_{-\theta^{\prime}}}<1$, for $\varepsilon>0$ sufficiently small, independent of $w$ and $w_{c s}$. Applying Lemma 2.5, we find that $w\left(w_{c s}\right)$ is $C^{1}$ from $\Sigma_{c s} \rightarrow \mathcal{B}_{-\theta^{\prime}}$ for any $\theta^{\prime}>0$ and $\varepsilon>0$ sufficiently small, with

$$
d w=\left(\operatorname{Id}-T_{w}\right)^{-1} T_{w_{c s}} .
$$

Differentiating (2.10) using the chain, product, and inverse linear operator derivative formulae, validated by the fact that $\left(\operatorname{Id}-T_{w}\right)^{-1}$ is uniformly bounded (since $\left|T_{w}\right| \leq \gamma<1$ ) from $\mathcal{B}_{-\theta^{\prime}} \rightarrow \mathcal{B}_{-\theta^{\prime}}$ for all $0 \leq \theta_{0}<\theta^{\prime}<\eta_{0}<\eta$, and the observation that higher (mixed) partial derivatives of $T$ exist and are continuous from $\mathcal{B}_{-\theta^{\prime} /(k+1)} \rightarrow \mathcal{B}_{-\theta^{\prime}}$ on the same range, we find that $w\left(w_{c s}\right)$ is $C^{k}$ from $\mathcal{B}_{-\tilde{\theta} / C} \rightarrow \mathcal{B}_{-\tilde{\theta}}$ for $C>0$ sufficiently large, so long as $\theta_{0}>0$ is chosen $\leq \tilde{\theta} / C$ and $\varepsilon>0$ is taken sufficiently small.

Finally, defining

$$
\Phi\left(w_{c s}\right):=\left.\Pi_{u} w\left(w_{s}\right)\right|_{t=0}=-\int_{0}^{+\infty} e^{A(t-s)} \Pi_{u} N(s, w(s)) d s,
$$

we obtain as claimed a $C^{k}$ function from $\Sigma_{c s} \rightarrow \Sigma_{u}$, whose graph is the invariant manifold of orbits growing at exponential rate $|w(t)| \leq C e^{\tilde{\theta} t}$ in forward time. From the latter characterization, we obtain evidently invariance in forward and backward time. By uniqueness of fixed point solutions, we have $w(0)=0$ and thus $\Phi_{c s}(0)=0$. Finally, differentiating (2.11) with respect to $w_{s}$, we obtain by Lemma 2.3

$$
d \Phi(0)=-\int_{0}^{+\infty} e^{A(t-s)} \Pi_{u} N_{w}(s, 0)\left(d w / d w_{s}\right)(s) d s=0
$$

since $N_{w}(0) \equiv 0$, yielding tangency as claimed.

\subsection{Local construction: proof of Theorem 2.1}

We can reduce the general situation, locally, to the case described in the global result by the following truncation procedure. Consider a general nonlinearity $N(t, w)$. Introducing a $C^{\infty}$ cutoff function

$$
\rho(x)= \begin{cases}1 & |x| \leq 1 \\ 0 & |x| \geq 2\end{cases}
$$


define $N^{\varepsilon}(t, w):=\rho(|w| / \varepsilon) N(t, w)$.

Lemma 2.7. Let $N \in C^{k+1}, k \geq 1$, and $N(t, 0) \equiv 0, \partial_{w} N(t, 0) \equiv 0$. Then, $N^{\varepsilon} \in C_{b}^{k+1}, N^{\varepsilon} \equiv N$ for $|u| \leq \varepsilon$, and the Lipschitz constant for $N^{\varepsilon}$ with respect to $w$ is uniformly bounded by $C \varepsilon$ for all $0<\varepsilon \leq \varepsilon_{0}$, where

$$
C=2\left(1+\max |r| \rho^{\prime}(|r|)\right) \max _{|w| \leq 2 \varepsilon_{0}}\left|\partial_{w}^{2} N(t, w)\right| .
$$

Proof. See Appendix A

Proof of Proposition 2.1. Defining $w:=u-u_{*}$, we obtain the nonlinear perturbation equation

$$
w^{\prime}=A w+N(w),
$$

with $A$ constant and $N \in C^{k+1}$ satisfying $N(0)=0, d N(0)=0$. Applying the truncation procedure, we obtain a modified equation

$$
w^{\prime}=A w+N^{\varepsilon}(w)
$$

for which $N^{\varepsilon} \in C_{b}^{k+1}$ with arbitrarily small Lipschitz norm $\varepsilon>0$ and $N \equiv N^{\varepsilon}$ within a neighborhood $B(0, \varepsilon)$ of $w=0$, i.e., with identical local flow. Applying Proposition 2.6, we obtain a global center stable manifold for (2.14), which is therefore a local center stable manifold for (2.13). Noting that solutions that stay uniformly bounded and close to the equilibrium for positive time are also bounded solutions of the truncated equations, we find that all such belong to the constructed center stable manifold.

Remark 2.8. The inclusion of $t$-dependence of $N$ in (2.8) is not needed for the present application (2.13), but allows also the treatment of time-periodic solutions after Floquet transformation to constant-coefficient linear part.

\section{Center Stable Manifold for PDE}

We now turn to the case of a general semilinear parabolic equation

$$
u_{t}=\mathcal{F}(u):=h\left(u, u_{x}\right)+u_{x x}, \quad u, h \in \mathbb{R}^{n},
$$

with steady state $u(x, t) \equiv \bar{u}(x)$ and associated group invariance

$$
\Psi_{\alpha}: \quad \Psi_{\alpha}(u)(x, t):=u(x+\alpha, t),
$$


and construct a local translation-invariant center stable manifold in the vicinity of $\bar{u}$.

Our approach follows closely that used to construct translation-invariant center manifolds in [TZ1], by introduction of a reduced flow on the quotient space induced by group equivalence. However, we coordinatize differently, using orthogonal projection rather than eigenprojections, to avoid the difficulty (as in the ultimate application to shock waves) that the zero eigenvale associated with translation-invariance may be embedded in essential spectrum of the linearized operator about the wave, as a consequence of which there may not exist a well-defined zero eigenprojection with respect to $H^{s}$ (see $[\mathrm{ZH}]$ for further discussion).

We make the following assumptions, in practice typically satisfied [TZ1].

(A0) $h \in C^{k+1}, k \geq 2$.

(A1) The linearized operator $L=\frac{\partial \mathcal{F}}{\partial u}(\bar{u})$ about $\bar{u}$ has $p$ unstable (positive real part) eigenvalues, with the rest of its spectrum of nonpositive real part.

(A2) $\left|\partial_{x}^{j} \bar{u}(x)\right| \leq C e^{-\theta|x|}, \theta>0$, for $1 \leq j \leq k+2$.

Proposition 3.1 (Center Stable Manifold Thm. for PDE). Under assumptions (AO)-(A2), there exists in an $H^{2}$ neighborhood of the set of translates of $\bar{u}$ a translation invariant $C^{k}$ (with respect to $H^{2}$ ) center stable manifold $\mathcal{M}_{c s}$, tangent at $\bar{u}$ to the center stable subspace $\Sigma_{c s}$ of $L$, that is (locally) invariant under the forward time-evolution of (3.1) and contains all solutions that remain bounded and sufficiently close to a translate of $\bar{u}$ in forward time. In general it is not unique.

\subsection{Reduced equations}

Differentiating with respect to $\alpha$ the relation $0 \equiv \partial_{t}\left(\Psi^{\alpha}(\bar{u})\right)=\mathcal{F}\left(\Psi^{\alpha}(\bar{u})\right)$, we recover the standard fact that

$$
\phi:={\frac{d \Psi_{\alpha}(\bar{u})}{d \alpha}}_{\mid \alpha=0}=\frac{\partial \bar{u}}{\partial x}
$$

is an $L^{2}$ zero eigenfunction of $L$, by the assumed decay of $\bar{u}_{x}$.

Define orthogonal projections

$$
\Pi_{2}:=\frac{\phi\langle\phi, \cdot\rangle}{|\phi|_{L^{2}}^{2}}, \quad \Pi_{1}:=\mathrm{Id}-\Pi_{2},
$$


onto the range of right zero-eigenfunction $\phi:=(\partial / \partial x) \bar{u}$ of $L$ and its orthogonal complement $\phi^{\perp}$ in $L^{2}$, where $\langle\cdot, \cdot\rangle$ denotes standard $L^{2}$ inner product.

Lemma 3.2. Under the assumed regularity $h \in C^{k+1}, k \geq 2, \Pi_{j}, j=1,2$ are bounded as operators from $H^{s}$ to itself for $0 \leq s \leq k+2$.

Proof. Immediate, by the assumed decay of $\phi=\bar{u}_{x}$ and derivatives.

Introducing the shifted perturbation variable

$$
v(x, t):=u(x+\alpha(t), t)-\bar{u}(x)
$$

similarly as in [Z4, MaZ2, TZ1], we obtain the nonlinear perturbation equation

$$
\partial_{t} v=L v+\mathcal{G}(v)-\partial_{t} \alpha\left(\phi+\partial_{x} v\right),
$$

where $L:=\frac{\partial \mathcal{F}}{\partial u}(\bar{u})$ and

$$
\mathcal{G}(v)=g\left(v, v_{x}, x\right):=h\left(\bar{u}+v, \bar{u}_{x}+v_{x}\right)-h\left(\bar{u}, \bar{u}_{x}\right)-d h\left(\bar{u}, \bar{u}_{x}\right)\left(v, v_{x}\right)
$$

is a quadratic-order Taylor remainder.

Choosing $\partial_{t} \alpha$ so as to cancel $\Pi_{2}$ of the righthand side of (3.5), we obtain finally the reduced equations

$$
\partial_{t} v=\Pi_{1}(L v+\mathcal{G}(v))
$$

and

$$
\partial_{t} \alpha=\frac{\pi_{2}(L v+\mathcal{G}(v))}{1+\pi_{2}\left(\partial_{x} v\right)}
$$

for $v \in \phi^{\perp}$, where $\pi_{2} v:=\langle\tilde{\phi}, v\rangle \|\left.\phi\right|_{L^{2}} ^{2}$, of the same regularity as the original equations.

Clearly, (3.8) is well-defined so long as $\left|\partial_{x} v\right|_{L^{\infty}} \leq C|v|_{H^{2}}$ remains small, hence we may solve the $v$ equation independently of $\alpha$, determining $\alpha$ behavior afterward to determine the full solution

$$
u(x, t)=\bar{u}(x-\alpha(t))+v(x-\alpha(t), t) .
$$


Moreover, it is easily seen (by the block-triangular structure of $L$ with respect to this decomposition) that the linear part $\Pi_{1} L=\Pi_{1} L \Pi_{1}$ of the $v$ equation possesses all spectrum of $L$ apart from the zero eigenvalue associated with eigenfunction $\phi$. Thus, we have effectively projected out this zero-eigenfunction, and with it the group symmetry of translation.

We may therefore construct the center stable manifold for the reduced equation (3.7), automatically obtaining translation-invariance when we extend to the full evolution using (3.8). See [TZ1] for further discussion.

\subsection{Preliminary estimates}

For ease of notation, introduce $L_{0}:=\Pi_{1} L, \mathcal{G}_{0}:=\Pi_{1} \mathcal{G}$.

Lemma 3.3. Under the assumed regularity $h \in C^{k+1}$, both $\mathcal{G}$ and $\mathcal{G}_{0}$ are Frechet differentiable of order $(k+1)$ considered respectively as functions from $H^{2}$ to $H^{1}$ and $\phi^{\perp} \subset H^{2}$ to $H^{1}: \mathcal{G}$ on the whole space and $\mathcal{G}_{0}$ for $|v|_{H^{2}}$ sufficiently small.

Proof. Differentiability of $\mathcal{G}$ follows by direct calculation; see [S, TZ1]. Differentiability of $\Pi_{1} \mathcal{G}$ follows similarly, using also the fact, already discussed, that $1+\pi_{2}\left(\partial_{x} v\right)$ remains bounded from zero for $|v|_{H^{2}}$ small, and the fact (see Lemma 3.2) that $\Pi_{j}$ as bounded linear operators from each $H^{s}$ to itself are infinitely differentiable in the Frechet sense.

Lemma 3.4. $L_{0}$ generates an analytic semigroup $e^{L_{0} t}=\Pi_{1} e^{L t} \Pi_{1}$ on $\phi^{\perp} \subset$ $H^{2}$. Moreover, the unstable (positive real part) spectra of $L$ and $L_{0}$ agree in both location and multiplicity, with associated total unstable eigenprojections $\Pi_{u}^{0}$ and $\Pi_{u}$ related by $\Pi_{u}^{0}=\Pi_{1} \Pi_{u} \Pi_{1}$ and total center stable eigenprojections $\Pi_{c s}^{0}$ and $\Pi_{c s}$ related by $\Pi_{c s}^{0}=\Pi_{1} \Pi_{c s} \Pi_{1}$. Likewise, except possibly at $\lambda=0$, the resolvent sets of $L$ and $L_{0}$ agree, with $\left(\lambda-L_{0}\right)^{-1}=\Pi_{1}(\lambda-L)^{-1} \Pi_{1}$.

Proof. Direct computation using relations $L \Pi_{1}=L$ and $L \Pi_{2}=0$ yields the resolvent relation, whence we obtain the remaining relations by their characterizations in terms of the resolvent (for example, the characterization of eigenprojection as residue of the resolvent operator [Kat]). As $L$ is a sectorial operator, it follows that $L_{0}$ is as well, and both generate analytic semigroups given by the inverse Laplace transform of the resolvent. 
Corollary 3.5 ([TZ1]). Under assumptions (A0)-(A2),

$$
\begin{aligned}
\left\|e^{t L_{0}} \Pi_{c s}\right\|_{H^{1} \rightarrow H^{2}} & \leq C_{\omega}\left(1+t^{-1 / 2}\right) e^{\omega t}, \\
\left\|e^{-t L_{0}} \Pi_{u}\right\|_{H^{1} \rightarrow H^{3}} & \leq C_{\omega} e^{-\beta t},
\end{aligned}
$$

for some $\beta>0$, and for all $\omega>0$, for all $t \geq 0$.

Proof. These follow from the corresponding estimates for $L$, which are standard semigroup estimates for second-order elliptic operators; see Appendix A.

Let $\rho$ be a smooth truncation function as in $\S 2.4$ and $\mathcal{G}_{0}^{\delta}(v):=\rho\left(\frac{|v|_{H^{2}}}{\delta}\right) \mathcal{G}_{0}(v)$.

Lemma 3.6 ([TZ1]). The map $\mathcal{G}_{0}^{\delta}: H^{2} \times \mathbb{R} \rightarrow H^{1} \times \mathbb{R}$ is $C^{k+1}$ and its Lipschitz norm with respect to $v$ is $O(\delta)$ as $\delta \rightarrow 0$.

Proof. See Appendix A.

Corollary 3.7. Under assumptions (A0)-(A2),

$$
\begin{aligned}
\left\|e^{t L_{0}} \Pi_{c s} \mathcal{G}_{0}^{\delta}\right\|_{H^{2} \rightarrow H^{2}} & \leq C_{\omega}\left(1+t^{-1 / 2}\right) e^{\omega t}, \\
\left\|e^{-t L_{0}} \Pi_{u} \mathcal{G}_{0}^{\delta}\right\|_{H^{2} \rightarrow H^{2}} & \leq C_{\omega} e^{-\beta t},
\end{aligned}
$$

for some $\beta>0$, and for all $\omega>0$, for all $t \geq 0$, with Lipshitz bounds

$$
\begin{aligned}
\left\|e^{t L_{0}} \Pi_{c s} d \mathcal{G}_{0}^{\delta}\right\|_{H^{2} \rightarrow H^{2}} & \leq C_{\omega} \delta\left(1+t^{-1 / 2}\right) e^{\omega t}, \\
\left\|e^{-t L_{0}} \Pi_{u} d \mathcal{G}_{0}^{\delta}\right\|_{H^{2} \rightarrow H^{2}} & \leq C_{\omega} \delta e^{-\beta t} .
\end{aligned}
$$

\subsection{Translation-invariant center stable manifold}

Proof of Proposition 3.1. Using bounds (3.9), (3.10), and (3.11), and observing that, since finite-dimensional, the unstable flow $e^{L_{0} t} \Pi_{u}$ is well-defined in both forward and backward time, we find, applying the finite-dimensional argument word-for-word, that the center stable manifold of the truncated flow $u_{t}=L_{0} u+\mathcal{G}_{0}^{\delta}(u)$ is the graph of the map $\Phi$ defined on $\tilde{\Sigma}_{c s}$ as $\Phi\left(u_{c s}\right)=\left(v_{c s}(0)\right)$, where $v_{c s}$ is the unique solution in $\phi^{\perp} \subset H^{2}$ of

$$
v(x, t)=e^{t L_{0}} u_{c s}+\int_{0}^{t} e^{(t-s) L_{0}} \Pi_{c s} \mathcal{G}_{0}^{\delta}(v(x, s)) d s-\int_{t}^{\infty} e^{(t-s) L_{0}} \Pi_{u} \mathcal{G}_{0}^{\delta}(v(x, s)) d s
$$

as guaranteed by the Contraction Mapping Theorem. 
Indeed, the only deviation from the finite-dimensional case is the appearance of the new factor $\left(1+(t-s)^{-1 / 2}\right)$ in the integrand of estimates having to do with integral term $\int_{0}^{t} e^{L_{0}(t-s)} \Pi_{c s} \mathcal{G}_{0}^{\delta}$, which, since integrable, does not alter the final estimates. The proof of smoothness relies on these same bounds, so likewise carries over word-for-word as in the finite-dimensional case. Finally, invariance in forward time follows by the characterization of the center stable manifold as the set of solutions of the truncated equations growing no faster than $|v(t)| \leq C e^{\beta t}$ in forward time. (Since the flow of (3.1) is only a semigroup, we cannot conclude invariance in backward time as in the finitedimensional case.) Since sufficiently small bounded solutions of the original are bounded solutions also of the truncated equations, they are contained in the center stable manifold, independent of the choice of truncation function.

This gives a center stable manifold with the stated properties for the reduced equation (3.7) for $v \in \phi^{\perp}$. Solving for the shift $\alpha$ in terms of $v$ using (3.8), and substituting $v, \alpha$ into (3.4) to obtain $u$, we obtain a translationinvariant center stable manifold for the original equations (3.1). Observing that small bounded solutions $v$ of the reduced equations, by (3.4), correspond to solutions $u$ of the (3.1) remaining close to a translate of $\bar{u}$, we are done.

\subsection{Application to viscous shock waves}

Proof of Theorem 1.1. By Proposition 3.1, it is sufficient to verify that (H0)(H1) imply (A0)-(A2), for $h\left(u, u_{x}\right):=f(u)_{x}=d f(u) u_{x}$. Clearly, (H0) implies (A0) by the form of $h$. Plugging $u=\bar{u}(x)$ into (1.1), we obtain the standingwave ODE $f(\bar{u})_{x}=\bar{u}_{x} x$, or, integrating from $-\infty$ to $x$, the first-order system

$$
\bar{u}_{x}=f(\bar{u})-f\left(u_{-}\right) .
$$

Linearizing about the assumed critical points $u_{ \pm}$yields linearized systems $w_{t}=d f\left(u_{ \pm}\right) w$, from which we see that $u_{ \pm}$are nondegenerate rest points by (H1), as a consequence of which (A2) follows by standard ODE theory [Co].

Finally, linearizing $\operatorname{PDE}(3.1)$ about the constant solutions $u \equiv u_{ \pm}$, we obtain $w_{t}=L_{ \pm} w:=-d f\left(u_{ \pm}\right) w_{x}-w_{x x}$. By Fourier transform, the limiting operators $L_{ \pm}$have spectra $\lambda_{j}^{ \pm}(k)=-i k a_{j}^{ \pm}(k)-k^{2}$, where the Fourier wavenumber $k$ runs over all of $\mathbb{R}$; in particular, $L_{ \pm}$have spectra of nonpositive real part. By a standard result of Henry [He], the essential spectrum of $L$ lies to the left of the rightmost boundary of the spectra of $L_{ \pm}$, hence we may conclude that the essential spectrum of $L$ is entirely nonpositive. As 
the spectra of $L$ to the right of the essential spectrum by sectoriality of $L$, consists of finitely many discrete eigenvalues, this means that the spectra of $L$ with positive real part consists of $p$ unstable eigenvalues, for some $p$, verifying (A1).

\section{Conditional stability analysis}

Define similarly as in Section 3.1 the perturbation variable

$$
v(x, t):=u(x+\alpha(t), t)-\bar{u}(x)
$$

for $u$ a solution of (1.1), where $\alpha$ is to be specified later in a way appropriate for the task at hand. Subtracting the equations for $u(x+\alpha(t), t)$ and $\bar{u}(x)$, we obtain the nonlinear perturbation equation

$$
v_{t}-L v=N(v)_{x}
$$

where $L:=-\partial_{x} d f(\bar{u})+\partial_{x}^{2}$ as in (1.2) denotes the linearized operator about $\bar{u}$ and

$$
N(v):=-(f(\bar{u}+v)-f(\bar{u})-d f(\bar{u}) v)
$$

where, so long as $|v|_{H^{1}}$ (hence $|v|_{L^{\infty}}$ and $|u|_{L^{\infty}}$ ) remains bounded,

$$
N(v)=O\left(|v|^{2}\right)
$$

\subsection{Projector bounds}

Let $\Pi_{u}$ denote the eigenprojection of $L$ onto its unstable subspace $\Sigma_{u}$, and $\Pi_{c s}=\mathrm{Id}-\Pi_{u}$ the eigenprojection onto its center stable subspace $\Sigma_{c s}$.

Lemma 4.1. Assuming (HO)-(H1),

$$
\Pi_{j} \partial_{x}=\partial_{x} \tilde{\Pi}_{j}
$$

for $j=u$, cs and, for all $1 \leq p \leq \infty, 0 \leq r \leq 4$,

$$
\begin{array}{r}
\left|\Pi_{c s}\right|_{W^{r, p} \rightarrow W^{r, p}},\left|\tilde{\Pi}_{c s}\right|_{W^{r, p} \rightarrow W^{r, p}} \leq C, \\
\left|\tilde{\Pi}_{c s}\right|_{W r, p \rightarrow W^{r, p}},\left|\tilde{\Pi}_{c s}\right|_{W r, p \rightarrow W^{r, p}} \leq C .
\end{array}
$$


Proof. Recalling (see the proof of Theorem 1.1) that $L$ has at most finitely many unstable eigenvalues, we find that $\Pi_{u}$ may be expressed as

$$
\Pi_{u} f=\sum_{j=1}^{p} \phi_{j}(x)\left\langle\tilde{\phi}_{j}, f\right\rangle
$$

where $\phi_{j}, j=1, \ldots p$ are generalized right eigenfunctions of $L$ associated with unstable eigenvalues $\lambda_{j}$, satisfying the generalized eigenvalue equation $\left(L-\lambda_{j}\right)^{r_{j}} \phi_{j}=0, r_{j} \geq 1$, and $\tilde{\phi}_{j}$ are generalized left eigenfunctions. Noting that $L$ is divergence form, and that $\lambda_{j} \neq 0$, we may integrate $\left(L-\lambda_{j}\right)^{r_{j}} \phi_{j}=0$ over $\mathbb{R}$ to obtain $\lambda_{j}^{r_{j}} \int \phi_{j} d x=0$ and thus $\int \phi_{j} d x=0$. Noting that $\phi_{j}, \tilde{\phi}_{j}$ and derivatives decay exponentially by standard theory [He, ZH, MaZ1], we find that

$$
\phi_{j}=\partial_{x} \Phi_{j}
$$

with $\Phi_{j}$ and derivatives exponentially decaying, hence

$$
\tilde{\Pi}_{u} f=\sum_{j} \Phi_{j}\left\langle\partial_{x} \tilde{\phi}, f\right\rangle .
$$

Estimating

$$
\left|\partial_{x}^{j} \Pi_{u} f\right|_{L^{p}}=\left|\sum_{j} \partial_{x}^{j} \phi_{j}\left\langle\tilde{\phi}_{j} f\right\rangle\right|_{L^{p}} \leq \sum_{j}\left|\partial_{x}^{j} \phi_{j}\right|_{L^{p}}\left|\tilde{\phi}_{j}\right|_{L^{q}}|f|_{L^{p}} \leq C|f|_{L^{p}}
$$

for $1 / p+1 / q=1$ and similarly for $\partial_{x}^{r} \tilde{\Pi}_{u} f$, we obtain the claimed bounds on $\Pi_{u}$ and $\tilde{\Pi}_{u}$, from which the bounds on $\Pi_{c s}=\mathrm{Id}-\Pi_{u}$ and $\tilde{\Pi}_{c s}=\mathrm{Id}-\tilde{\Pi}_{u}$ follow immediately.

\subsection{Linear estimates}

Let $G_{c s}(x, t ; y):=\Pi_{c s} e^{L t} \delta_{y}(x)$ denote the Green kernel of the linearized solution operator on the center stable subspace $\Sigma_{c s}$. Then, we have the following detailed pointwise bounds established in [TZ2, MaZ1].

Proposition 4.2 ([TZ2, MaZ1]). Assuming (H0)-(H2), (D1)-D(3), the center stable Green function may be decomposed as $G_{c s}=E+\tilde{G}$, where

$$
E(x, t ; y)=\partial_{x} \bar{u}(x) e_{j}(y, t)
$$




$$
e(y, t)=\sum_{a_{k}^{-}>0}\left(\operatorname{errfn}\left(\frac{y+a_{k}^{-} t}{\sqrt{4 t}}\right)-\operatorname{errfn}\left(\frac{y-a_{k}^{-} t}{\sqrt{4 t}}\right)\right) l_{k}^{-}(y)
$$

for $y \leq 0$ and symmetrically for $y \geq 0, l_{k}^{-} \in \mathbb{R}^{n}$ constant, and

$$
\begin{aligned}
|\tilde{G}(x, t ; y)| \leq & C e^{-\eta(|x-y|+t)}+\sum_{k=1}^{n} t^{-1 / 2} e^{-\left(x-y-a_{k}^{-} t\right)^{2} / M t} e^{-\eta x^{+}} \\
& +\sum_{a_{k}^{-}>0, a_{j}^{-}<0} \chi_{\left\{\left|a_{k}^{-} t\right| \geq|y|\right\}} t^{-1 / 2} e^{-\left(x-a_{j}^{-}\left(t-\left|y / a_{k}^{-}\right|\right)\right)^{2} / M t} e^{-\eta x^{+}} \\
& +\sum_{a_{k}^{-}>0, a_{j}^{+}>0} \chi_{\left\{\left|a_{k}^{-} t\right| \geq|y|\right\}} t^{-1 / 2} e^{-\left(x-a_{j}^{+}\left(t-\left|y / a_{k}^{-}\right|\right)\right)^{2} / M t} e^{-\eta x^{-}},
\end{aligned}
$$

$$
\begin{aligned}
\left|\partial_{y} \tilde{G}(x, t ; y)\right| \leq & C e^{-\eta(|x-y|+t)}+C t^{-1 / 2}\left(\sum_{k=1}^{n} t^{-1 / 2} e^{-\left(x-y-a_{k}^{-} t\right)^{2} / M t} e^{-\eta x^{+}}\right. \\
& +\sum_{a_{k}^{-}>0, a_{j}^{-}<0} \chi_{\left\{\left|a_{k}^{-} t\right| \geq|y|\right\}} t^{-1 / 2} e^{-\left(x-a_{j}^{-}\left(t-\left|y / a_{k}^{-}\right|\right)\right)^{2} / M t} e^{-\eta x^{+}} \\
& \left.+\sum_{a_{k}^{-}>0, a_{j}^{+}>0} \chi_{\left\{\left|a_{k}^{-} t\right| \geq|y|\right\}} t^{-1 / 2} e^{-\left(x-a_{j}^{+}\left(t-\left|y / a_{k}^{-}\right|\right)\right)^{2} / M t} e^{-\eta x^{-}}\right)
\end{aligned}
$$

for $y \leq 0$ and symmetrically for $y \geq 0$, for some $\eta, C, M>0$, where $a_{j}^{ \pm}$ are the eigenvalues of $d f\left(u_{ \pm}\right), x^{ \pm}$denotes the positive/negative part of $x$, and indicator function $\chi_{\left\{\left|a_{k}^{-} t\right| \geq|y|\right\}}$ is 1 for $\left|a_{k}^{-} t\right| \geq|y|$ and 0 otherwise.

Proof. As observed in [TZ2], it is equivalent to establish decomposition

$$
G=G_{u}+E+\tilde{G}
$$

for the full Green function $G(x, t ; y):=e^{L t} \delta_{y}(x)$, where

$$
G_{u}(x, t ; y):=\Pi_{u} e^{L t} \delta_{y}(x)=e^{\gamma t} \sum_{j=1}^{p} \phi_{j}(x) \tilde{\phi}_{j}(y)^{t}
$$

for some constant matrix $M \in \mathbb{C}^{p \times p}$ denotes the Green kernel of the linearized solution operator on $\Sigma_{u}, \phi_{j}$ and $\tilde{\phi}_{j}$ right and left generalized eigenfunctions associated with unstable eigenvalues $\lambda_{j}, j=1, \ldots, p$. 
The problem of describing the full Green function has been treated in [ZH, MaZ3], starting with the Inverse Laplace Transform representation

$$
G(x, t ; y)=e^{L t} \delta_{y}(x)=\oint_{\Gamma} e^{\lambda t}(\lambda-L(\varepsilon))^{-1} \delta_{y}(x) d \lambda,
$$

where

$$
\Gamma:=\partial\left\{\lambda: \Re \lambda \leq \eta_{1}-\eta_{2}|\Im \lambda|\right\}
$$

is an appropriate sectorial contour, $\eta_{1}, \eta_{2}>0$; estimating the resolvent kernel $G_{\lambda}^{\varepsilon}(x, y):=(\lambda-L(\varepsilon))^{-1} \delta_{y}(x)$ using Taylor expansion in $\lambda$, asymptotic ODE techniques in $x, y$, and judicious decomposition into various scattering, excited, and residual modes; then, finally, estimating the contribution of various modes to (4.12) by Riemann saddlepoint (Stationary Phase) method, moving contour $\Gamma$ to a optimal, "minimax" positions for each mode, depending on the values of $(x, y, t)$.

In the present case, we may first move $\Gamma$ to a contour $\Gamma^{\prime}$ enclosing (to the left) all spectra of $L$ except for the $p$ unstable eigenvalues $\lambda_{j}, j=1, \ldots, p$, to obtain

$$
G(x, t ; y)=\oint_{\Gamma^{\prime}} e^{\lambda t}(\lambda-L)^{-1} d \lambda+\sum_{j= \pm} \operatorname{Residue}_{\lambda_{j}(\varepsilon)}\left(e^{\lambda t}(\lambda-L)^{-1} \delta_{y}(x)\right)
$$

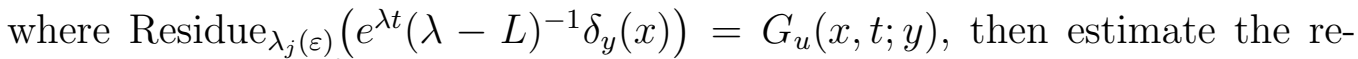
maining term $\oint_{\Gamma^{\prime}} e^{\lambda t}(\lambda-L)^{-1} d \lambda$ on minimax contours as just described. See the proof of Proposition 7.1, [MaZ3], for a detailed discussion of minimax estimates $E+G$ and of Proposition 7.7, [MaZ3] for a complementary discussion of residues incurred at eigenvalues in $\{\Re \lambda \geq 0\} \backslash\{0\}$. See also [TZ1].

Corollary 4.3 ([MaZ1]). Assuming (HO)-(H2), (D1)-(D3),

$$
\begin{gathered}
\left|\int_{-\infty}^{+\infty} \tilde{G}(\cdot, t ; y) f(y) d y\right|_{L^{p}} \leq C(1+t)^{-\frac{1}{2}\left(\frac{1}{q}-\frac{1}{p}\right)}|f|_{L^{q}}, \\
\left|\int_{-\infty}^{+\infty} \tilde{G}_{y}(\cdot, t ; y) f(y) d y\right|_{L^{p}} \leq C(1+t)^{-\frac{1}{2}\left(\frac{1}{q}-\frac{1}{p}\right)-\frac{1}{2}}|f|_{L^{q}},
\end{gathered}
$$

for all $t \geq 0$, some $C>0$, for any $1 \leq q \leq p$ (equivalently, $1 \leq r \leq p$ ) and $f \in L^{q}$, where $1 / r+1 / q=1+1 / p$. 
Proof. Standard convolution inequalities together with bounds (4.9)-(4.10); see [MaZ1, MaZ2, MaZ3, Z2] for further details.

Corollary 4.4 ([Z4]). The kernel e satisfies

$$
\begin{gathered}
\left|e_{y}(\cdot, t)\right|_{L^{p}},\left|e_{t}(\cdot, t)\right|_{L^{p}} \leq C t^{-\frac{1}{2}(1-1 / p)}, \\
\left|e_{t y}(\cdot, t)\right|_{L^{p}} \leq C t^{-\frac{1}{2}(1-1 / p)-1 / 2},
\end{gathered}
$$

for all $t>0$. Moreover, for $y \leq 0$ we have the pointwise bounds

$$
\begin{gathered}
\left|e_{y}(y, t)\right|,\left|e_{t}(y, t)\right| \leq C t^{-\frac{1}{2}} \sum_{a_{k}^{-}>0}\left(e^{-\frac{\left(y+a_{k}^{-} t\right)^{2}}{M t}}+e^{-\frac{\left(y-a_{k}^{-} t\right)^{2}}{M t}}\right), \\
\left|e_{t y}(y, t)\right| \leq C t^{-1} \sum_{a_{k}^{-}>0}\left(e^{-\frac{\left(y+a_{k}^{-} t\right)^{2}}{M t}}+e^{-\frac{\left(y-a_{k}^{-} t\right)^{2}}{M t}}\right),
\end{gathered}
$$

for $M>0$ sufficiently large, and symmetrically for $y \geq 0$.

Proof. Direct computation using with definition (4.8); see Appendix A.

\subsection{Reduced equations II}

Recalling that $\partial_{x} \bar{u}$ is a stationary solution of the linearized equations $u_{t}=L u$, so that $L \partial_{x}=0$, or

$$
\int_{-\infty}^{\infty} G(x, t ; y) \bar{u}_{x}(y) d y=e^{L t} \bar{u}_{x}(x)=\partial_{x} \bar{u}(x),
$$

we have, applying Duhamel's principle to (4.2),

$$
\begin{aligned}
v(x, t) & =\int_{-\infty}^{\infty} G(x, t ; y) v_{0}(y) d y \\
& -\int_{0}^{t} \int_{-\infty}^{\infty} G_{y}(x, t-s ; y)(N(v)+\dot{\alpha} v)(y, s) d y d s+\alpha(t) \partial_{x} \bar{u}(x) .
\end{aligned}
$$

Defining

$$
\begin{aligned}
\alpha(t) & =-\int_{-\infty}^{\infty} e(y, t) v_{0}(y) d y \\
& +\int_{0}^{t} \int_{-\infty}^{+\infty} e_{y}(y, t-s)(N(v)+\dot{\alpha} v)(y, s) d y d s
\end{aligned}
$$


following [ZH, Z4, MaZ2, MaZ3], where $e$ is defined as in (4.8), and recalling the decomposition $G=E+G_{u}+\tilde{G}$ of (4.11), we obtain the reduced equations

$$
\begin{aligned}
v(x, t) & =\int_{-\infty}^{\infty}\left(G_{u}+\tilde{G}\right)(x, t ; y) v_{0}(y) d y \\
& -\int_{0}^{t} \int_{-\infty}^{\infty}\left(G_{u}+\tilde{G}\right)_{y}(x, t-s ; y)(N(v)+\dot{\alpha} v)(y, s) d y d s,
\end{aligned}
$$

and, differentiating (4.15) with respect to $t$, and observing that $e_{y}(y, s) \rightarrow 0$ as $s \rightarrow 0$, as the difference of approaching heat kernels,

$$
\begin{aligned}
\dot{\alpha}(t) & =-\int_{-\infty}^{\infty} e_{t}(y, t) v_{0}(y) d y \\
& +\int_{0}^{t} \int_{-\infty}^{+\infty} e_{y t}(y, t-s)(N(v)+\dot{\alpha} v)(y, s) d y d s .
\end{aligned}
$$

We emphasize that this (nonlocal in time) choice of $\alpha$ and the resulting reduced equations are completely different from those of Section 3.1, according to their respective purposes. A third possible choice has been introduced in [GMWZ1, GMWZ2] for the study of the inviscid limit problem. As discussed further in [Go, Z4, MaZ2, MaZ3, GMWZ1, BeSZ], $\alpha$ may be considered in the present context as defining a notion of approximate shock location.

\subsection{Nonlinear damping estimate}

Proposition 4.5 ([MaZ3]). Assuming (HO)-(H3), let $v_{0} \in H^{4}$, and suppose that for $0 \leq t \leq T$, the $H^{4}$ norm of $v$ remains bounded by a sufficiently small constant, for $v$ as in (4.1) and $u$ a solution of (1.1). Then, for some constants $\theta_{1,2}>0$, for all $0 \leq t \leq T$,

$$
\|v(t)\|_{H^{4}}^{2} \leq C e^{-\theta_{1} t}\|v(0)\|_{H^{4}}^{2}+C \int_{0}^{t} e^{-\theta_{2}(t-s)}\left(|v|_{L^{2}}^{2}+|\dot{\alpha}|^{2}\right)(s) d s .
$$

Proof. Subtracting the equations for $u(x+\alpha(t), t)$ and $\bar{u}(x)$, we may write the perturbation equation for $v$ alternatively as

$$
v_{t}+\left(\int_{0}^{1} d f(\bar{u}(x)+\tau v(x, t)) d \tau v\right)_{x}-v_{x x}=\dot{\alpha}(t) \partial_{x} \bar{u}(x) .
$$


Observing that $\partial_{x}^{j}\left(\partial_{x} \bar{u}\right)(x)=O\left(e^{-\eta|x|}\right)$ is bounded in $L^{1}$ norm for $j \leq 4$, we take the $L^{2}$ inner product in $x$ of $\sum_{j=0}^{4} \partial_{x}^{2 j} v$ against (4.19), integrate by parts and rearrange the resulting terms to arrive at the inequality

$$
\partial_{t}\|v\|_{H^{4}}^{2}(t) \leq-\theta\left\|\partial_{x}^{5} v\right\|_{L^{2}}^{2}+C\left(\|v\|_{H^{4}}^{2}+|\dot{\alpha}(t)|^{2}\right),
$$

$\theta>0$, for $C>0$ sufficiently large, so long as $\|v\|_{H^{4}}$ remains bounded. Using the Sobolev interpolation

$$
\|v\|_{H^{4}}^{2} \leq \tilde{C}^{-1}\left\|\partial_{x}^{5} v\right\|_{L^{2}}^{2}+\tilde{C}\|v\|_{L^{2}}^{2}
$$

for $\tilde{C}>0$ sufficiently large, we obtain

$$
\partial_{t}\|v\|_{H^{4}}^{2}(t) \leq-\tilde{\theta}\|v\|_{H^{4}}^{2}+C\left(\|v\|_{L^{2}}^{2}+|\dot{\alpha}(t)|^{2}\right),
$$

from which (4.18) follows by Gronwall's inequality.

\subsection{Proof of nonlinear stability}

Decompose now the nonlinear perturbation $v$ as

$$
v(x, t)=w(x, t)+z(x, t),
$$

where

$$
w:=\Pi_{c s} v, \quad z:=\Pi_{u} v
$$

Applying $\Pi_{c s}$ to (4.16) and recalling commutator relation (4.5), we obtain an equation

$$
\begin{aligned}
w(x, t)= & \int_{-\infty}^{\infty} \tilde{G}(x, t ; y) w_{0}(y) d y \\
& -\int_{0}^{t} \int_{-\infty}^{\infty} \tilde{G}_{y}(x, t-s ; y) \tilde{\Pi}_{c s}(N(v)+\dot{\alpha} v)(y, s) d y d s
\end{aligned}
$$

for the flow along the center stable manifold, parametrized by $w \in \Sigma_{c s}$.

Lemma 4.6. Assuming (HO)-(H1), for v lying initially on the center stable manifold $\mathcal{M}_{c s}$

$$
|z|_{W^{r, p}} \leq C|w|_{H^{2}}^{2}
$$

for some $C>0$, for all $1 \leq p \leq \infty$ and $0 \leq r \leq 4$, so long as $|w|_{H^{2}}$ remains sufficiently small. 
Proof. By tangency of the center stable manifold to $\Sigma_{c s}$, we have immediately $|z|_{H^{2}} \leq C|w|_{H^{2}}^{2}$, whence (4.23) follows by equivalence of norms for finite-dimensional vector spaces, applied to the $p$-dimensional subspace $\Sigma_{u}$. (Alternatively, we may see this by direct computation using the explicit description of $\Pi_{u} v$ afforded by Lemma 4.1.)

Proof of Theorem 1.2. Recalling by Theorem 1.1 that solutions remaining for all time in a sufficiently small radius neighborhood $\mathcal{N}$ of the set of translates of $\bar{u}$ lie in the center stable manifold $\mathcal{M}_{c s}$, we obtain trivially that solutions not originating in $\mathcal{M}_{c s}$ must exit $\mathcal{N}$ in finite time, verifying the final assertion of orbital instability with respect to perturbations not in $\mathcal{M}_{c s}$.

Consider now a solution $v \in \mathcal{M}_{c s}$, or, equivalently, a solution $w \in \Sigma_{c s}$ of (4.22) with $z=\Phi_{c s}(w) \in \Sigma_{u}$. Define

$$
\zeta(t):=\sup _{0 \leq s \leq t}\left(|w|_{H^{2}}(1+s)^{\frac{1}{4}}+|w|_{L^{\infty}}+|\dot{\alpha}(s)|(1+s)^{\frac{1}{2}}\right) .
$$

We shall establish:

Claim. For all $t \geq 0$ for which a solution exists with $\zeta$ uniformly bounded by some fixed, sufficiently small constant, there holds

$$
\zeta(t) \leq C_{2}\left(E_{0}+\zeta(t)^{2}\right) \text { for } \quad E_{0}:=\left|v_{0}\right|_{L^{1} \cap H^{2}} .
$$

From this result, provided $E_{0}<1 / 4 C_{2}^{2}$, we have that $\zeta(t) \leq 2 C_{2} E_{0}$ implies $\zeta(t)<2 C_{2} E_{0}$, and so we may conclude by continuous induction that

$$
\zeta(t)<2 C_{2} E_{0}
$$

for all $t \geq 0$, whence we obtain the stated bounds by definition (4.24). (By Lemma 4.5 and standard short-time $H^{s}$ existence theory, $v \in H^{4}$ exists and $\zeta$ remains continuous so long as $\zeta$ remains bounded by some uniform constant, hence (4.26) is an open condition.) Thus, it remains only to establish the claim above.

Proof of Claim. We must show that $u\left(\theta+\psi_{1}+\psi_{2}\right)^{-1}$ and $|\dot{\alpha}(s)|(1+s)$ are each bounded by $C\left(E_{0}+\zeta(t)^{2}\right)$, for some $C>0$, all $0 \leq s \leq t$, so long as $\zeta$ remains sufficiently small.

By Lemma 4.6, $\left|w_{0}\right|_{L^{1} \cap H^{2}} \leq\left|v_{0}\right|_{L^{1} \cap H^{2}}+\left|z_{0}\right|_{L^{1} \cap H^{2}} \leq\left|v_{0}\right|_{L^{1} \cap H^{2}}+C\left|w_{0}\right|_{H^{2}}^{2}$, whence

$$
\left|w_{0}\right|_{L^{1} \cap H^{2}} \leq C E_{0}
$$


Likewise, by Lemma 4.6, (4.24), (4.4), and Lemma 4.1, for $0 \leq s \leq t$,

$$
\left|\tilde{\Pi}_{c s}(N(v)+\dot{\alpha} v)(y, s)\right|_{L^{q}} \leq C \zeta(t)^{2}(1+s)^{-\frac{5}{4}} .
$$

Combining the latter bounds with representations (4.22)-(4.17), taking $q=2$ in (4.27), and applying Corollary 4.3, we obtain

$$
\begin{aligned}
|w(x, t)|_{L^{p}} \leq & \left|\int_{-\infty}^{\infty} \tilde{G}(x, t ; y) w_{0}(y) d y\right|_{L^{p}} \\
& +\left|\int_{0}^{t} \int_{-\infty}^{\infty} \tilde{G}_{y}(x, t-s ; y) \tilde{\Pi}_{c s}(N(v)+\dot{\alpha} v)(y, s) d y d s\right|_{L^{p}} \\
\leq & E_{0}(1+t)^{-\frac{1}{2}\left(1-\frac{1}{p}\right)} \\
& +C \zeta(t)^{2} \int_{0}^{t} \int_{-\infty}^{\infty}(t-s)^{-\frac{3}{4}+\frac{1}{2 p}}(1+s)^{-\frac{3}{4}} d y d s \\
\leq & C\left(E_{0}+\zeta(t)^{2}\right)(1+t)^{-\frac{1}{2}\left(1-\frac{1}{p}\right)}
\end{aligned}
$$

and, similarly, using Hölder's inequality and applying Corollary 4.4,

$$
\begin{aligned}
|\dot{\alpha}(t)| \leq & \int_{-\infty}^{\infty}\left|e_{t}(y, t)\right|\left|v_{0}(y)\right| d y \\
& \quad+\int_{0}^{t} \int_{-\infty}^{+\infty}\left|e_{y t}(y, t-s)\right|\left|\tilde{\Pi}_{c s}(N(v)+\dot{\alpha} v)(y, s)\right| d y d s \\
\leq & \left|e_{t}\right|_{L^{\infty}}\left|v_{0}\right|_{L^{1}}+C \zeta(t)^{2} \int_{0}^{t}\left|e_{y t}\right|_{L^{2}}(t-s)\left|\tilde{\Pi}_{c s}(N(v)+\dot{\alpha} v)\right|_{L^{2}}(s) d s \\
\leq & E_{0}(1+t)^{-\frac{1}{2}}+C \zeta(t)^{2} \int_{0}^{t}(t-s)^{-\frac{3}{4}}(1+s)^{-\frac{3}{4}} d s \\
\leq & C\left(E_{0}+\zeta(t)^{2}\right)(1+t)^{-\frac{1}{2}} .
\end{aligned}
$$

Applying Lemma 4.5 and using (4.28) and (4.29), we obtain, finally,

$$
|w|_{H^{2}}(t) \leq C\left(E_{0}+\zeta(t)^{2}\right)(1+t)^{-\frac{1}{4}} .
$$

Combining (4.28), (4.29), and (4.30), we obtain (4.25) as claimed, completing the proof of the Claim and the theorem.

Remark 4.7. We point out that the finite-dimensional part $z$ of $v$ is in fact controlled pointwise by its $L^{2}$ norm and thus by $|w|_{H^{2}}$, satisfying $|z(x, t)| \leq$ 
$C e^{-\theta|x|}|z(\cdot, t)|_{L^{2}(x)} \leq C e^{-\theta|x|}|w(\cdot, t)|_{H^{2}(x)}^{2}$, making possible a pointwise version of the argument above. This is a key point in treating the nonclassical over- or undercompressive cases, which appear to require pointwise bounds [HZ, RZ].

Acknowledgement. Thanks to Milena Stanislavova and Charles Li for two interesting discussions that inspired this work, and to Milena Stanislavova for pointing out the reference in [GJLS].

\section{A Proofs of miscellaneous lemmas}

We include for completeness the proofs of earlier cited lemmas that were not proved in the main body of the text.

Proof of Lemma 2.2. By Taylor's Theorem,

$$
g\left(f_{2}(t)\right)-g\left(f_{1}(t)\right)=d g\left(f_{1}(t)\right)\left(f_{2}(t)-f_{1}(t)\right)+o\left(\left|f_{2}(t)-f_{1}(t)\right|\right),
$$

as $\left|f_{2}(t)-f_{1}(t)\right| \rightarrow 0$. By the assumed uniform boundedness of $|d g|$, we readily obtain that $f \rightarrow d g f$ is a bounded linear operator from $\mathcal{B}_{\eta} \rightarrow \mathcal{B}_{\eta}$. On the other hand $\left|f_{2}-f_{1}\right|(t) \leq e^{-\eta\left(t-t_{0}\right)}\left\|f_{2}-f_{1}\right\|_{\eta}$ for $t \geq t_{0}$ implies $\left|f_{2}-f_{1}\right| \rightarrow 0$ uniformly on $t \geq t_{0}$ as $\left\|f_{2}-f_{1}\right\|_{\eta} \rightarrow 0$, and so we have

$$
\begin{aligned}
\| g\left(f_{2}(t)\right)-g\left(f_{1}(t)\right)- & d g\left(f_{1}(t)\right)\left(f_{2}(t)-f_{1}(t)\right) \|_{\eta}= \\
& \sup _{t \geq t_{0}} e^{\eta\left(t-t_{0}\right)} o\left(\left|f_{2}(t)-f_{1}(t)\right|\right)=o\left(\left\|f_{2}-f_{1}\right\|_{\eta},\right.
\end{aligned}
$$

yielding the result for $k=1$. The general result then follows by induction on $k$, applying the result for $k=1$ to successively higher derivatives of $g$.

Proof of Lemma 2.4. (i) Triangulating, we have

$$
\begin{aligned}
\left|y\left(x_{2}\right)-y\left(x_{1}\right)\right| & =\mid T\left(x_{2}, y\left(x_{2}\right)\right)-T\left(x_{1}, y\left(x_{1}\right) \mid\right. \\
& \leq \mid T\left(x_{2}, y\left(x_{2}\right)\right)-T\left(x_{2}, y\left(x_{1}\right)|+| T\left(x_{2}, y\left(x_{1}\right)\right)-T\left(x_{1}, y\left(x_{1}\right) \mid\right.\right. \\
& \leq \theta\left|y\left(x_{2}\right)-y\left(x_{1}\right)\right|+L\left|x_{2}-x_{1}\right|
\end{aligned}
$$

where $0<\theta<1$ and $0<L$ are contraction and Lipschitz coefficients, yielding after rearrangement $\left|y\left(x_{2}\right)-y\left(x_{1}\right)\right| \leq \frac{L}{1-\theta}\left|x_{2}-x_{1}\right|$.

(ii) Applying Taylor's Theorem, and using the result of (i), we have (A.3)

$$
\begin{aligned}
y\left(x_{2}\right)-y\left(x_{1}\right) & =T_{x}\left(x_{2}-x_{1}\right)+T_{y}\left(y_{2}-y_{1}\right)+o\left(\left|x_{2}-x_{1}\right|+\left|y\left(x_{2}\right)-y\left(x_{1}\right)\right|\right) \\
& =T_{x}\left(x_{2}-x_{1}\right)+T_{y}\left(y_{2}-y_{1}\right)+o\left(\left|x_{2}-x_{1}\right|\right),
\end{aligned}
$$


where all derivatives are evaluated at $\left(x_{1}, y\left(x_{1}\right)\right)$. Noting that the operator norm $\left|T_{y}\right|$ is bounded by contraction coefficient $0<\theta<1$, we have by Neumann series expansion that $\left(\mathrm{Id}-T_{y}\right)$ is invertible with uniformly bounded inverse $\left|\left(\operatorname{Id}-T_{y}\right)^{-1}\right| \leq(1-\theta)^{-1}$. Thus, rearranging, we have

$$
y\left(x_{2}\right)-y\left(x_{1}\right)=\left(\operatorname{Id}-T_{y}\right)^{-1} T_{x}\left(x_{2}-x_{1}\right)+o\left(\left|x_{2}-x_{1}\right|\right),
$$

yielding the result for $k=1$ by definition of (Frechet) derivative. The results for $k \geq 1$ then follow by induction upon differentiation of (2.6).

Proof of Lemma 2.7. The Lipshitz constant is bounded by $\max \left|\partial_{w} N^{\varepsilon}\right|$, where

$$
\begin{aligned}
\left|\partial_{w} N^{\varepsilon}\right| & =\left|\left(\rho^{\varepsilon}\right)^{\prime} N+\rho^{\varepsilon} \partial_{w} N\right| \\
& =\left|(|w| / \varepsilon) \rho^{\prime}(|w| / \varepsilon)(N(t, w) /|w|)+\rho(|w| / \varepsilon) \partial_{w} N(t, w)\right| \\
& \leq 2 \varepsilon\left(\max |r| \rho^{\prime}(|r|) \max _{|w| \leq 2 \varepsilon_{0}} \frac{|N(t, w)|}{|w|^{2}}+\max _{|w| \leq 2 \varepsilon_{0}} \frac{\left|\partial_{w} N(t, w)\right|}{|w|}\right) \\
& \leq 2 \varepsilon\left(\max |r| \rho^{\prime}(|r|) \max _{|w| \leq 2 \varepsilon_{0}}+1\right) \max _{|w| \leq 2 \varepsilon_{0}}\left|\partial_{w}^{2} N(t, w)\right|
\end{aligned}
$$

the final inequality following by $N(t, 0) \equiv 0, \partial_{w} N(t, 0) \equiv 0$ and the Integral Mean Value Theorem, or first-order Taylor remainder formula.

Proof of Corollary 3.5. By Lemma 3.4, it is sufficient to prove the corresponding bounds for the purely differential operator $L$. By sectoriality of $L$, we have the inverse Laplace transform representations

$$
\begin{aligned}
e^{t L} \Pi_{u} & :=\int_{\Gamma_{u}} e^{\lambda t}(\lambda-L)^{-1} d \lambda, \\
e^{t L} \Pi_{c s} & :=\int_{\Gamma_{c s}} e^{\lambda t}(\lambda-L)^{-1} d \lambda,
\end{aligned}
$$

where $\Gamma_{c s}$ denotes a sectorial contour bounding the center and stable spectrum to the right $[\mathrm{Pa}]$, which by (A1) may be taken so that $\Re \Gamma_{s} \leq \omega$, and $\Gamma_{u}$ denotes a closed curve enclosing the unstable spectrum of $L$, with $\Re \Gamma_{s} \geq \beta>0$.

Applying the resolvent formula $L(\lambda-L)^{-1}=\lambda(\lambda-L)^{-1}-I d$, we obtain in the standard way

$$
e^{t L} \Pi_{j}:=\int_{\Gamma_{j}} \lambda e^{\lambda t}(\lambda-L)^{-1} d \lambda,
$$


from which we obtain immediately the second stated bound, and, by a scaling argument $[\mathrm{Pa}]$, the bound

$$
\left\|e^{t L} \Pi_{c s}\right\|_{H^{1} \rightarrow H^{3}} \leq\left\|L e^{t L} \Pi_{s}\right\|_{H^{1} \rightarrow H^{1}} \leq C\left(1+t^{-1}\right) e^{\omega t} .
$$

Recalling the standard bound $\left\|e^{t L} \prod_{c s}\right\|_{H^{1} \rightarrow H^{1}} \leq C e^{\omega t}$, and interpolating between $|\cdot|_{H^{1}}$ and $|\cdot|_{H^{3}}$, we obtain the first stated bound.

Proof of Lemma 3.6. The norm in $H^{2}$ is a quadratic form, hence the map

$$
v \in H^{2} \mapsto \rho\left(\frac{|v|_{H^{2}}}{\delta}\right) \in \mathbb{R}_{+},
$$

is smooth, and $\mathcal{G}_{0}^{\delta}$ is as regular as $\mathcal{G}_{0}$. Now

$$
\begin{aligned}
\left|\mathcal{G}_{0}^{\delta}\left(v_{1}\right)-\mathcal{G}_{0}^{\delta}\left(v_{2}\right)\right|_{H^{1}} \leq & \left|\rho\left(\frac{\left|v_{1}\right|_{H^{2}}}{\delta}\right)-\rho\left(\frac{\left|v_{2}\right|_{H^{2}}}{\delta}\right)\right|_{L^{\infty}}\left|\mathcal{G}_{0}\left(v_{1}\right)\right|_{H^{1}} \\
& +\left|\rho\left(\frac{\left|v_{2}\right|_{H^{2}}}{\delta}\right)\right|_{L^{\infty}}\left|\mathcal{G}_{0}\left(v_{1}\right)-\mathcal{G}_{0}\left(v_{2}\right)\right|_{H^{1}} \\
\leq & 3\left|v_{1}-v_{2}\right|_{H^{2}}\left(\sup _{|v|_{H^{2}}<\delta} \frac{\left|\mathcal{G}_{0}(v)\right|_{H^{1}}}{\delta}+\sup _{|v|_{H^{2}}<\delta}\left|d \mathcal{G}_{0}(v)\right|_{H^{1}}\right),
\end{aligned}
$$

and $\sup _{|v|_{H^{2}}<\delta}\left|\mathcal{G}_{0}(v)\right|_{H^{1}}=O\left(\delta^{2}\right), \sup _{|v|_{H^{2}}<\delta}\left|d \mathcal{G}_{0}(v)\right|_{H^{1}}=O(\delta)$.

Proof of Corollary 4.4. For definiteness, take $y \leq 0$. Then, (4.8) gives

$$
\begin{gathered}
e_{y}(y, t):=\sum_{a_{k}^{-}>0}\left[c_{k,-}^{0}\right] l_{k}^{-t}\left(K\left(y+a_{k}^{-} t, t\right)-K\left(y-a_{k}^{-} t, t\right)\right) \\
e_{t}(y, t):=\sum_{a_{k}^{-}>0}\left[c_{k,-}^{0}\right] l_{k}^{-t}\left(\left(K+K_{y}\right)\left(y+a_{k}^{-} t, t\right)-\left(K+K_{y}\right)\left(y-a_{-} k^{t}, t\right)\right), \\
e_{t y}(y, t):=\sum_{a_{k}^{-}>0}\left[c_{k,-}^{0}\right] l_{k}^{-t}\left(\left(K_{y}+K_{y y}\right)\left(y+a_{k}^{-} t, t\right)-\left(K_{y}+K_{y y}\right)\left(y-a_{k}^{-} t, t\right)\right),
\end{gathered}
$$

where

$$
K(y, t):=\frac{e^{-y^{2} / 4 t}}{\sqrt{4 \pi t}}
$$


denotes the standard heat kernel. The pointwise bounds (4.4)-(4.4) follow immediately for $t \geq 1$ by properties of the heat kernel, in turn yielding (4.4)(4.4) in this case. The bounds for small time $t \leq 1$ follow from estimates

$$
\begin{aligned}
\left|K_{y}(y+a t, t, \beta)-K_{y}(y-a t, t, \beta)\right| & =\left|\int_{y+a t}^{y-a t} K_{y y}(z, t, \beta) d z\right| \\
& \leq C t^{-3 / 2} \int_{y+a t}^{y-a t} e^{\frac{-z^{2}}{M t}} d z \leq C t^{-1 / 2} e^{-\frac{y^{2}}{M t}},
\end{aligned}
$$

and, similarly,

$$
\begin{aligned}
\left|K_{y y}(y+a t, t, \beta)-K_{y y}(y-a, t, \beta)\right| & =\left|\int_{-a t}^{a t} K_{y y y}(z, t, \beta) d z\right| \\
& \leq C t^{-2} \int_{y+a t}^{y-a t} e^{\frac{-z^{2}}{M t}} d z \leq C t^{-1} e^{-\frac{y^{2}}{M t}} .
\end{aligned}
$$

The bounds for $\left|e_{y}\right|$ are again immediate.

\section{References}

[AGJ] J. Alexander, R. Gardner and C.K.R.T. Jones, A topological invariant arising in the analysis of traveling waves, J. Reine Angew. Math. 410 (1990) 167-212.

[AMPZ] A. Azevedo-D. Marchesin-B. Plohr-K. Zumbrun, Nonuniqueness of solutions of Riemann problems. Z. Angew. Math. Phys. 47 (1996), 977-998.

[B] A. Bressan, A tutorial on the center manifold theorem,, Appendix A, "Hyperbolic Systems of Balance Laws," Lecture Notes in Mathematics 1911, Springer-Verlag, 2007.

[BeSZ] M. Beck, B. Sandstede, and K. Zumbrun, Nonlinear stability of time-periodic shock waves, preprint (2008).

[Co] W.A. Coppel, Stability and asymptotic behavior of differential equations, D.C. Heath and Co., Boston, MA (1965) viii+166 pp.

[GMWZ1] Gues, O., Metivier, G., Williams, M., and Zumbrun, K., Existence and stability of multidimensional shock fronts in the vanishing viscosity limit, Arch. Ration. Mech. Anal. 175 (2005), no. 2, 151-244.

[GMWZ2] Gues, O., Metivier, G., Williams, M., and Zumbrun, K., Paper 4, NavierStokes regularization of multidimensional Euler shocks, Ann. Sci. École Norm. Sup. (4) 39 (2006), no. 1, 75-175. 
[Go] J. Goodman, Remarks on the stability of viscous shock waves, in: Viscous profiles and numerical methods for shock waves (Raleigh, NC, 1990), 66-72, SIAM, Philadelphia, PA, (1991).

[GZ] R. Gardner and K. Zumbrun, The Gap Lemma and geometric criteria for instability of viscous shock profiles.

[He] D. Henry, Geometric theory of semilinear parabolic equations. Lecture Notes in Mathematics, Springer-Verlag, Berlin (1981), iv + 348 pp.

[HZ] P. Howard and K. Zumbrun, Stability of undercompressive viscous shock waves, in press, J. Differential Equations 225 (2006), no. 1, 308-360.

[Kat] T. Kato, Perturbation theory for linear operators. Springer-Verlag, Berlin Heidelberg (1985).

[GJLS] F. Gesztesy, C.K.R.T. Jones, Y. Latushkin, and M. Stanislavova, M., A spectral mapping theorem and invariant manifolds for nonlinear Schrdinger equations, Indiana Univ. Math. J. 49 (2000), no. 1, 221-243.

[Li] C. Li, On the Dynamics of Navier-Stokes and Euler Equations, Preprint, (2006).

[MM] J. E. Marsden, M. McCracken, The Hopf bifurcation and its applications, Applied Mathematical Sciences 19, Springer Verlag, New York, 1976.

[MaZ1] C. Mascia and K. Zumbrun, Pointwise Green function bounds for shock profiles of systems with real viscosity. Arch. Ration. Mech. Anal. 169 (2003), no. $3,177-263$.

[MaZ2] C. Mascia and K. Zumbrun, Stability of small-amplitude shock profiles of symmetric hyperbolic-parabolic systems, Comm. Pure Appl. Math. 57 (2004), no. $7,841-876$.

[MaZ3] C. Mascia and K. Zumbrun, Stability of large-amplitude viscous shock profiles of hyperbolic-parabolic systems. Arch. Ration. Mech. Anal. 172 (2004), no. 1, 93-131.

[Pa] A. Pazy, Semigroups of linear operators and applications to partial differential equations. Applied Mathematical Sciences, 44, Springer-Verlag, New YorkBerlin, (1983) viii+279 pp. ISBN: 0-387-90845-5.

[PW] R. L. Pego-M.I. Weinstein, Asymptotic stability of solitary waves, Comm. Math. Phys. 164 (1994), no. 2, 305-349.

[RZ] M. Raoofi and K. Zumbrun, Stability of undercompressive viscous shock profiles of hyperbolic-parabolic systems, Preprint (2007). 
[SS] B. Sandstede and A. Scheel, Hopf bifurcation from viscous shock waves, Preprint; received November 22, 2006.

[S] D. Sattinger, On the stability of waves of nonlinear parabolic systems. Adv. Math. 22 (1976) 312-355.

[TZ1] B. Texier and K. Zumbrun, Relative Poincaré-Hopf bifurcation and galloping instability of traveling waves, Methods Anal. and Appl. 12 (2005), no. 4, 349380 .

[TZ2] B. Texier and K. Zumbrun, Galloping instability of viscous shock waves, to appear, Physica D (2008).

[TZ3] B. Texier and K. Zumbrun, Hopf bifurcation of viscous shock waves in gas dynamics and MHD, to appear, Archive for Rat. Mech. Anal. (2008).

[TZ4] B. Texier and K. Zumbrun, Transition to longitudinal instability of detonation waves is generically associated with Hopf bifurcation to time-periodic galloping solutions, preprint (2008).

[VI] A. Vanderbauwhede and G. Iooss, Center manifold theory in infinite dimensions, Dynamics reported: expositions in dynamical systems, 125-163, Dynam. Report. Expositions Dynam. Systems (N.S.), 1, Springer, Berlin, 1992.

[Z1] K. Zumbrun, Multidimensional stability of planar viscous shock waves, Advances in the theory of shock waves, 307-516, Progr. Nonlinear Differential Equations Appl., 47, Birkhäuser Boston, Boston, MA, 2001.

[Z2] K. Zumbrun, Stability of large-amplitude shock waves of compressible NavierStokes equations, in Handbook of Mathematical Fluid Dynamics, Elsevier (2004).

[Z3] K. Zumbrun, Planar stability criteria for viscous shock waves of systems with real viscosity, in Hyperbolic Systems of Balance Laws, CIME School lectures notes, P. Marcati ed., Lecture Note in Mathematics 1911, Springer (2004).

[Z4] K. Zumbrun, Refined Wave-tracking and Nonlinear Stability of Viscous Lax Shocks. Methods Appl. Anal. 7 (2000) 747-768.

[ZH] K. Zumbrun and P. Howard, Pointwise semigroup methods and stability of viscous shock waves. Indiana Univ. Math. J. 47 (1998), 741-871; Errata, Indiana Univ. Math. J. 51 (2002), no. 4, 1017-1021.

[ZS] K. Zumbrun and D. Serre, Viscous and inviscid stability of multidimensional planar shock fronts, Indiana Univ. Math. J. 48 (1999) 937-992. 